\title{
Global existence and exponential stability for the micropolar fluid system
}

\author{
E. J. Villamizar-Roa* \& M. A. Rodríguez-Bellido ${ }^{\ddagger}$
}

April 20, 2007

\begin{abstract}
We consider the micropolar fluid system in a bounded domain of $\mathbb{R}^{3}$ and prove the existence and the uniqueness of a global strong solution with initial data being a perturbation of the stationary solution, whose existence is also obtained. We prove that these solutions converge uniformly to the stationary solutions with exponential decay rate. The technique of our analysis is the semigroups approach in $L^{p}$-spaces.
\end{abstract}

Keywords: Micropolar fluids, strong solution, exponential decay rate, semigroup approach.

AMS subject classification: 35A, 35B35, 35B40, 35B65, 35Q35, 76D03

\section{Introduction}

In this paper we study the following equations for the motion of micropolar fluid in a bounded domain $\Omega \subset \mathbb{R}^{3}$ with smooth boundary $\partial \Omega$ :

$$
\left\{\begin{array}{c}
\partial_{t} \boldsymbol{u}+(\boldsymbol{u} \cdot \nabla) \boldsymbol{u}-\left(\mu+\mu_{r}\right) \Delta \boldsymbol{u}+\nabla p=2 \mu_{r} \operatorname{rot} \boldsymbol{w}+\boldsymbol{f}, \quad \text { in } \Omega \times(0, \infty), \\
\partial_{t} \boldsymbol{w}+(\boldsymbol{u} \cdot \nabla) \boldsymbol{w}-\chi \Delta \boldsymbol{w}-\gamma \nabla \operatorname{div} \boldsymbol{w}+4 \mu_{r} \boldsymbol{w}=2 \mu_{r} \operatorname{rot} \boldsymbol{u}+\boldsymbol{g}, \quad \text { in } \Omega \times(0, \infty), \\
\operatorname{div} \boldsymbol{u}=0, \quad \text { in } \Omega \times(0, \infty),
\end{array}\right.
$$

where the unknowns are $\boldsymbol{u}=\boldsymbol{u}(\mathbf{x}, t) \in \mathbb{R}^{3}, \boldsymbol{w}=\boldsymbol{w}(\mathbf{x}, t) \in \mathbb{R}^{3}$ and $p=p(\mathbf{x}, t) \in$ $\mathbb{R}$ which denote respectively, the linear velocity, the velocity of rotation of the fluid particles and the pressure, at a point $(\mathbf{x}, t) \in \Omega \times(0, \infty)$. The terms $\boldsymbol{f}(\mathbf{x}, t)$ and $\boldsymbol{g}(\mathbf{x}, t)$ are given, and stand external sources of the linear and angular momentum of particles, respectively. $\mu, \mu_{r}, c_{0}, c_{a}, c_{d}$ are positive constants given to characterize isotropic properties of the fluid: $\mu$ is the usual Newtonian viscosity; $\mu_{r}, c_{0}, c_{a}, c_{d}$ are new viscosities related to the asymmetry of the stress

*E. J. Villamizar-Roa is supported by Colciencias, Colombia, Proyecto BID-III etapa and Universidad Industrial de Santander-UIS.

${ }^{\dagger}$ M. A. Rodríguez-Bellido is partially supported by D.G.E.S. and M.C. y T. (Spain), Projet BFM2003-06446-C02-01 and by M.E.C. (Spain), Projet MTM2006-07932.

$\ddagger$ Corresponding author 
tensor and consequently related to the appearance of the internal rotation field $\boldsymbol{w}$. In order to shorten the equations, we denote $\chi=c_{a}+c_{d}$ and $\gamma=c_{0}+c_{d}-c_{a}$. These constants satisfy $c_{0}+c_{d}>c_{a}$. We use the standard notations $\nabla, \Delta$, div and rot for the gradient, Laplacian, divergence and rotational operators respectively, and $\partial_{t} \boldsymbol{u}$ to denote the time derivative. The $i^{t h}$ component of $(\boldsymbol{u} \cdot \nabla) \boldsymbol{w}$ in cartesian coordinates is given by $[(\boldsymbol{u} \cdot \nabla) \boldsymbol{w}]_{i}=\sum_{j=1}^{3} u_{j} \frac{\partial w_{i}}{\partial x_{j}}$. Together with these equations we prescribe the following initial and boundary conditions

$$
\begin{gathered}
\boldsymbol{u}(\mathbf{x}, 0)=\boldsymbol{u}_{0}(\mathbf{x}), \quad \boldsymbol{w}(\mathbf{x}, 0)=\boldsymbol{w}_{0}(\mathbf{x}), \quad \text { in } \Omega, \\
\boldsymbol{u}(\mathbf{x}, t)=\mathbf{0}, \quad \boldsymbol{w}(\mathbf{x}, t)=\mathbf{0}, \quad \text { on } \partial \Omega \times(0, \infty),
\end{gathered}
$$

where, for the sake of simplicity in this exposition, we have taken homogeneous boundary conditions for both velocity and microrotational fields.

The formulation and motivation of the equations (1)-(3) are found in Petrosyan [25], Condiff \& Dalher [3], Eringen [4, 5] and Lukaszewicz [21], that treat the magneto-micropolar model which can be particularized to the micropolar model case. We observe that this model includes as a particular case the classical Navier-Stokes equations, which has been greatly studied (see the classical books by Ladyzhenskaya [17]; Temam [30] and the references therein).

With respect to system (1)-(3), Lukaszewicz [19, 20] established the global existence of weak solutions and local strong solutions for (1)-(3) using linearization and an almost fixed point theorem. Using the spectral Galerkin method: Boldrini \& Rojas-Medar [26] proved the global existence of weak solutions; the local strong solution was obtained by Rojas-Medar [27], and the global one by Ortega-Torres \& Rojas-Medar [22]. The convergence rates by the spectral Galerkin method were established in Rojas-Medar [28]. Another work that showed the existence and uniqueness of strong solutions (local in time for any data and global in time for small data) is Ortega-Torres \& Rojas-Medar [23]. However, those works were made only in the Hilbertian context. Recently, Yamaguchi [31] studied the problem (1)-(3) in a bounded domain using the semigroup approach in $L^{p}, 1<p<\infty$, showing the existence of global strong solutions for small data.

Here, we study the global existence, asymptotic behavior and stability of the solutions for the micropolar fluid system. If we consider smallness conditions on the physical constants of the system, we prove the global existence of strong solutions for system (1)-(3) in different spaces for the linear and angular velocity (the equal case was considered by Yamaguchi [31]). In a second step, we show that such solutions converge uniformly to stationary solutions when $t \rightarrow \infty$ with exponential decay rate. Thus, we say that these stationary solutions are asymptotically stable. We consider the initial data $\left(\boldsymbol{u}_{0}, \boldsymbol{w}_{0}\right) \in \mathbf{L}^{3}(\Omega) \times \mathbf{L}^{m}(\Omega)$, for $m \geq 3 / 2$. In particular, we show the existence of strong solutions in the space $\mathbf{L}^{3}(\Omega) \times \mathbf{L}^{3}(\Omega)$.

To show the existence of stationary solutions for system (1)-(3) in the space $\mathbf{L}^{3}(\Omega) \times \mathbf{L}^{m}(\Omega)$, we use a fixed point argument. On the other hand, we obtain estimates of type $\left(\mathbf{L}^{p} \times \mathbf{L}^{q}\right)-\left(\mathbf{L}^{r} \times \mathbf{L}^{s}\right)$ for the analytic semigroup generated by 
the linearization of the operator in a neighborhood of the stationary solution, which include the cases $p \neq q$ and $r \neq s$, and consequently we prove the global existence and asymptotic behavior of the solutions of the system (1)-(3). In this sense, our results on the global existence generalize the results that Yamaguchi [31] studied for the initial value problem, only for the cases $p=q$ and $r=s$. The existence of the semigroup is obtained through the estimates $\left(\mathbf{L}^{p} \times \mathbf{L}^{q}\right)-$ $\left(\mathbf{L}^{r} \times \mathbf{L}^{s}\right)$ for the resolvent of the linearized operator, whose behavior depends on exponents $p, q, r, s$.

From the stability point of view, several papers are devoted to study of the stability of the stationary solutions for the particular case of the Navier-Stokes equations, see for instance, Kozono \& Ozawa [15], Borchers \& Miyakawa [1], Chen [2], Kozono \& Ogawa [14], Kozono \& Yamazaki [16]. In general, these studies have been made using a characterization of the fractional power of the associated linearized operator and estimates of kind $L^{p}-L^{r}$ for the semigroup. On the other hand, in the convection problem, which is a model of the hydrodynamic formed by a coupling between the Navier-Stokes equations with a equation for the temperature, Hishida $[11,12]$ analyzed the stability problem in bounded and exterior domains, in the framework of $L^{p}$ and weak- $L^{p}$ spaces, by means of $\mathbf{L}^{p}-\mathbf{L}^{r}$ and weak- $\mathbf{L}^{p}-\mathbf{L}^{r}$ estimates for the semigroup associated with the respective linearized operator. The works cited before motivated our analysis about the stability for the micropolar fluid system.

By sections, this paper is organized as follows: In $\S 2$ we give some preliminary results, some definitions and state our principal results. In $\S 3$, we study the existence of a steady solution. The linearized operator is analyzed at $\S 4$. Finally, in $\S 5$ we prove the global existence of nonstationary solutions near a stationary solution and show that the nonstationary solution tends to the stationary solution as $t \rightarrow \infty$ with a exponential rate.

\section{Preliminaries and main results}

Let $\Omega$ be a bounded domain of $\mathbb{R}^{3}$ with smooth boundary $\partial \Omega$. We consider the usual Sobolev spaces $W^{m, q}(\Omega)=\left\{f \in L^{q}(\Omega):\left\|\partial^{k} f\right\|_{L^{q}(\Omega)}<\infty\right.$, for $\left.|k| \leq m\right\}$, for $m \geq 1$ and $1 \leq q \leq \infty$ with the norm denoted by $\|\cdot\|_{m, q}$. As it is usual, we denote by $W_{0}^{1, p}(\Omega)$ the closure of $C_{0}^{\infty}(\Omega)$ (functions of class $C^{\infty}(\Omega)$ with compact support) in $W^{1, p}(\Omega)$. We denote by $\|\cdot\|_{\{p, q\}}$ the norm in $L^{p}(\Omega) \times L^{q}(\Omega)$ and by $\|\cdot\|_{\{p, q\} \rightarrow\{r, s\}}$ (respectively, $\|\cdot\|_{p \rightarrow r}$ ) the norm of a bounded operator of $L^{p}(\Omega) \times L^{q}(\Omega) \rightarrow L^{r}(\Omega) \times L^{s}(\Omega)$ (respectively, of $L^{p}(\Omega) \rightarrow L^{r}(\Omega)$ ). We denote by $C_{0, \sigma}^{\infty}$ the space of the functions $C_{0}^{\infty}$ with free divergence and by $L_{\sigma}^{p}(\Omega), 1<p<\infty$, the closure of $C_{0, \sigma}^{\infty}$ in the $L^{p}(\Omega)$-norm. When the functions to be vectors, the space will appear in bold characters.

It is well known the Helmholtz decomposition of vectorial field in $\mathbf{L}^{p}$ (see for instance Fujiwara \& Morimoto [6]) $\mathbf{L}^{p}(\Omega)=\mathbf{L}_{\sigma}^{p}(\Omega) \oplus\left\{\nabla q: q \in W^{1, p}(\Omega)\right\}$. Let $P=P_{p}$ be the projector of $\mathbf{L}^{p}(\Omega)$ in $\mathbf{L}_{\sigma}^{p}(\Omega)$. Then, the Stokes operator in 
$\mathbf{L}_{\sigma}^{p}(\Omega), 1<p<\infty$, is given by

$$
D\left(A_{p}\right)=\mathbf{L}_{\sigma}^{p}(\Omega) \cap \mathbf{W}_{0}^{1, p}(\Omega) \cap \mathbf{W}^{2, p}(\Omega), \quad A_{p} \boldsymbol{u}=-P_{p} \Delta \boldsymbol{u} .
$$

Also, we denote the Laplace operator in $\mathbf{L}^{q}(\Omega), 1<q<\infty$, by

$$
D\left(B_{q}\right)=\mathbf{W}_{0}^{1, q}(\Omega) \cap \mathbf{W}^{2, q}(\Omega), \quad B_{q} \mathbf{v}=-\Delta \mathbf{v} .
$$

It is well known that the Stokes operator generates a bounded analytic semigroup $\left\{e^{-t A_{p}}\right\}_{t \geq 0}$, of class $C_{0}$ in $\mathbf{L}_{\sigma}^{p}(\Omega), 1<p<\infty$, see Giga [8]). It is also a classical result the Laplace operator generates a bounded analytic semigroup $\left\{e^{-t B_{q}}\right\}_{t \geq 0}$ of class $C_{0}$ in $\mathbf{L}^{q}(\Omega), 1<q<\infty$. From now on, we sometimes drop the subscripts $p$ and $q$ attached to operators $A_{p}, B_{q}$ if there is no confusion.

We will consider the following class of stationary solutions for the problem (1)-(3)

Definition 2.1 Let $3 / 2 \leq m<\infty$ and $K>0$. We say that a pair of functions $(\overline{\boldsymbol{u}}, \overline{\boldsymbol{w}})$ is a stationary solution of class $(m, K)$ for (1)-(3), if

$$
(\overline{\boldsymbol{u}}, \overline{\boldsymbol{w}}) \in D\left(A_{3}\right) \times D\left(B_{m}\right),\|A \overline{\boldsymbol{u}}\|_{3}+\|B \overline{\boldsymbol{w}}\|_{m} \leq K,
$$

and $(\overline{\boldsymbol{u}}, \overline{\boldsymbol{w}})$ satisfies

$$
\left\{\begin{aligned}
\left(\mu+\mu_{r}\right) A \overline{\boldsymbol{u}}-2 \mu_{r} \operatorname{rot} \overline{\boldsymbol{w}}+P\{(\overline{\boldsymbol{u}} \cdot \nabla) \overline{\boldsymbol{u}}\}=0, & \text { in } \mathbf{L}_{\sigma}^{3}(\Omega), \\
\chi B \overline{\boldsymbol{w}}+(\overline{\boldsymbol{u}} \cdot \nabla) \overline{\boldsymbol{w}}-\gamma \nabla \operatorname{div} \overline{\boldsymbol{w}}+4 \mu_{r} \overline{\boldsymbol{w}}-2 \mu_{r} \operatorname{rot} \overline{\boldsymbol{u}}=0, & \text { in } \mathbf{L}^{m}(\Omega) .
\end{aligned}\right.
$$

Here, we have used that $\mathbf{W}^{1, m}(\Omega) \subset \mathbf{L}^{3}(\Omega)$ together with the fact that $P(\operatorname{rot} \overline{\boldsymbol{w}})=$ $\operatorname{rot} \overline{\boldsymbol{w}}$. We observe that $\operatorname{div} \operatorname{rot} \overline{\boldsymbol{w}}=0$ in $\Omega$; and therefore, since $\overline{\boldsymbol{w}}=0$ on $\partial \Omega$, we have $\operatorname{rot} \overline{\boldsymbol{w}} \cdot \mathbf{n}=0$ on $\partial \Omega$. Thus, $\operatorname{rot} \overline{\boldsymbol{w}} \in \mathbf{L}_{\sigma}^{3}(\Omega)$. Moreover, we observe that $\mathbf{W}^{1,3}(\Omega) \subset \mathbf{L}^{m}(\Omega)$.

The following theorem states the existence of the stationary solution of class $(m, K)$.

Theorem 2.2 For each $m \in[3 / 2, \infty)$ there exists $\bar{\delta}=\bar{\delta}(m)$ and $K_{0}>0$ such that if $\mu, \chi \geq \bar{\delta}$, then the problem (1)-(3) has a unique stationary solution $(\overline{\boldsymbol{u}}, \overline{\boldsymbol{w}})$ of class $\left(m, K_{0}\right)$.

Remark 2.1 The existence of stationary solutions in bounded domains was studied by Lukaszewicz [18] (see also Lukaszewicz [21]). The proof was done using the Leray-Schauder's Theorem. The existence of weak solutions is showed considering two problems, a problem for $\boldsymbol{u}$, with $\boldsymbol{w}$ fixed and later, a problem for $\boldsymbol{w}$ with $\boldsymbol{u}$ given. Under smallness conditions on the viscosity, the solution is unique. Regularity results can also be found. More precisely, the solution obtained by Lukaszewicz [21] belongs to $\mathbf{V} \times \mathbf{H}_{0}^{1}(\Omega)$ (when weak solutions are considered) and the space $\mathbf{V} \times\left(\mathbf{H}_{0}^{1}(\Omega) \cap \mathbf{H}^{2}(\Omega)\right.$ ) (when strong solutions are considered). We remark that $\mathbf{V}=\left\{\mathbf{v} \in \mathbf{H}_{0}^{1}(\Omega)\right.$, $\left.\operatorname{div} \mathbf{v}=0\right\}$. 
In order to establish the stability of the stationary solutions $(\overline{\boldsymbol{u}}, \overline{\boldsymbol{w}})$ given by Theorem 2.2, and give a global strong solution of (1)-(3), we make $\boldsymbol{u}=\overline{\boldsymbol{u}}+$ $\widetilde{\boldsymbol{u}}, \boldsymbol{w}=\overline{\boldsymbol{w}}+\widetilde{\boldsymbol{w}}, p=\bar{p}+\widetilde{p}$, in (1)-(3), where $\bar{p}$ is the associated pressure with $(\overline{\boldsymbol{u}}, \overline{\boldsymbol{w}})$ and $(\widetilde{\boldsymbol{u}}, \widetilde{\boldsymbol{w}}, \widetilde{p})$ denotes the perturbation of $(\overline{\boldsymbol{u}}, \overline{\boldsymbol{w}}, \bar{p})$.

Definition 2.3 A pair of functions $(\boldsymbol{u}, \boldsymbol{w})$ is a strong solution of (1)-(3) on $[0, \infty)$ if the perturbation $(\widetilde{\boldsymbol{u}}, \widetilde{\boldsymbol{w}})$ belongs to the class

$$
\begin{aligned}
& \widetilde{\boldsymbol{u}}=\boldsymbol{u}-\overline{\boldsymbol{u}} \in C\left([0, \infty) ; \mathbf{L}_{\sigma}^{3}(\Omega)\right) \cap C\left((0, \infty) ; D\left(A_{3}\right)\right) \cap C^{1}\left((0, \infty) ; \mathbf{L}_{\sigma}^{3}(\Omega)\right), \\
& \widetilde{\boldsymbol{w}}=\boldsymbol{w}-\overline{\boldsymbol{w}} \in C\left([0, \infty) ; \mathbf{L}^{m}(\Omega)\right) \cap C\left((0, \infty) ; D\left(B_{m}\right)\right) \cap C^{1}\left((0, \infty) ; \mathbf{L}^{m}(\Omega)\right),
\end{aligned}
$$

for $m \in[3 / 2, \infty)$, and satisfies

$$
\left\{\begin{array}{rr}
\partial_{t} \widetilde{\boldsymbol{u}}+\left(\mu+\mu_{r}\right) A \widetilde{\boldsymbol{u}}+P\{(\widetilde{\boldsymbol{u}} \cdot \nabla) \widetilde{\boldsymbol{u}}+(\overline{\boldsymbol{u}} \cdot \nabla) \widetilde{\boldsymbol{u}}+(\widetilde{\boldsymbol{u}} \cdot \nabla) \overline{\boldsymbol{u}}\}- & \\
-2 \mu_{r} \operatorname{rot} \widetilde{\boldsymbol{w}}=0, & t>0, \\
\partial_{t} \widetilde{\boldsymbol{w}}+\chi B \widetilde{\boldsymbol{w}}+(\widetilde{\boldsymbol{u}} \cdot \nabla) \widetilde{\boldsymbol{w}}+(\overline{\boldsymbol{u}} \cdot \nabla) \widetilde{\boldsymbol{w}}+(\widetilde{\boldsymbol{u}} \cdot \nabla) \overline{\boldsymbol{w}} & \\
-\gamma \nabla \operatorname{div} \widetilde{\boldsymbol{w}}+4 \mu_{r} \widetilde{\boldsymbol{w}}-2 \mu_{r} \operatorname{rot} \overline{\boldsymbol{u}}=0, & t>0, \\
\overline{\boldsymbol{u}}(0)=\boldsymbol{u}_{0}-\overline{\boldsymbol{u}}, & \widetilde{\boldsymbol{w}}(0)=\boldsymbol{w}_{0}-\overline{\boldsymbol{w}} .
\end{array}\right.
$$

Our result on stability of stationary solution is the following.

Theorem 2.4 Let $3 / 2 \leq m<\infty$ and assume $\left(\boldsymbol{u}_{0}, \boldsymbol{w}_{0}\right) \in \mathbf{L}_{\sigma}^{3}(\Omega) \times \mathbf{L}^{m}(\Omega)$. Then, there exists $\delta \in(0, \bar{\delta}]$ (where $\bar{\delta}$ is from Theorem 2.2) such that if $\gamma+\mu_{r}<\delta$, $\max \left\{\frac{1}{\mu+\mu_{r}}, \frac{1}{\chi}\right\}<\delta$, then the stationary solution $(\overline{\boldsymbol{u}}, \overline{\boldsymbol{w}})$ given by Theorem 2.2 is stable, that is, there exists a positive constant $\epsilon=\epsilon\left(\mu, \mu_{r}, \chi, \gamma, m\right)$ such that if

$$
\left\|\boldsymbol{u}_{0}-\overline{\boldsymbol{u}}\right\|_{3}+\left\|\boldsymbol{w}_{0}-\overline{\boldsymbol{w}}\right\|_{m}<\epsilon,
$$

then the system (1)-(3) has a unique strong solution $(\boldsymbol{u}, \boldsymbol{w})$ on $[0, \infty)$. Moreover, this solution decays exponentially, that is, $\|\boldsymbol{u}(t)-\overline{\boldsymbol{u}}\|_{\infty}+\|\boldsymbol{w}(t)-\overline{\boldsymbol{w}}\|_{\infty}=o\left(e^{-\zeta t}\right)$, when $t \rightarrow \infty$, with $\zeta=\zeta\left(\mu, \mu_{r}, \chi, \gamma, m\right)$ some positive constant.

\section{Proof of Theorem 2.2}

In this section we build a stationary solution $(\overline{\boldsymbol{u}}, \overline{\boldsymbol{w}})$ of class $(m, K)$. To this aim, we will use Banach's Fixed Point Theorem. Thus, we focus our efforts in the building of a contractive operator $F$. This operator is defined as $F$ : $D\left(A_{3}\right) \times D\left(B_{m}\right) \rightarrow D\left(A_{3}\right) \times D\left(B_{m}\right)$ is given by:

$$
F\left[\begin{array}{c}
\overline{\boldsymbol{u}} \\
\overline{\boldsymbol{w}}
\end{array}\right]=\left[\begin{array}{c}
-\frac{1}{\mu+\mu_{r}} A^{-1}\left\{P\{(\overline{\boldsymbol{u}} \cdot \nabla) \overline{\boldsymbol{u}}\}-2 \mu_{r} \operatorname{rot} \overline{\boldsymbol{w}}\right\} \\
-\frac{1}{\chi} B^{-1}\left\{(\overline{\boldsymbol{u}} \cdot \nabla) \overline{\boldsymbol{w}}+4 \mu_{r} \overline{\boldsymbol{w}}-\gamma \nabla \operatorname{div} \overline{\boldsymbol{w}}-2 \mu_{r} \operatorname{rot} \overline{\boldsymbol{u}}\right\}
\end{array}\right] .
$$

We observe that the system (4) is equivalent to

$$
[\overline{\boldsymbol{u}}, \overline{\boldsymbol{w}}]^{T}=F[\overline{\boldsymbol{u}}, \overline{\boldsymbol{w}}]^{T}, \quad \text { in } D\left(A_{3}\right) \times D\left(B_{m}\right) .
$$

Hence, the proof of Theorem 2.2 is a consequence of the following proposition. 
Proposition 3.1 Let $m \in[3 / 2, \infty)$. There are positive constants $\bar{\delta}=\bar{\delta}(m)$ and $K_{0}=K_{0}\left(\mu, \mu_{r}, \chi, \gamma, m\right)>0$ such that if $\mu, \chi \geq \bar{\delta}$, then the operator $F$ is contractive on the complete metric space $E_{K_{0}}=\left\{(\overline{\boldsymbol{u}}, \overline{\boldsymbol{w}}) \in D\left(A_{3}\right) \times D\left(B_{m}\right)\right.$ : $\left.\|A \overline{\boldsymbol{u}}\|_{3}+\|B \overline{\boldsymbol{w}}\|_{m} \leq K_{0}\right\}$.

To prove Proposition 3.1 we will use the following lemma, which follows from embedding properties obtained by Giga [10], the Hölder's inequality and the Sobolev's embedding.

Lemma 3.2 Let $1<p, q, r<\infty$ be satisfying the relations

$$
\frac{1}{r}>\frac{1}{p}-\frac{2}{3}, \frac{1}{r}>\frac{1}{q}-\frac{1}{3}, \frac{1}{r} \geq \frac{1}{p}+\frac{1}{q}-1 .
$$

Then, the following estimates hold: for all $\boldsymbol{u} \in D\left(A_{p}\right), \boldsymbol{v} \in D\left(A_{q}\right), \boldsymbol{w} \in D\left(B_{q}\right)$

$$
\begin{gathered}
\|P\{(\boldsymbol{u} \cdot \nabla) \boldsymbol{v}\}\|_{r} \leq C(p, q, r)\|A \boldsymbol{u}\|_{p}\|A \boldsymbol{v}\|_{q}, \\
\|(\boldsymbol{u} \cdot \nabla) \boldsymbol{w}\|_{r} \leq C(p, q, r)\|A \boldsymbol{u}\|_{p}\|B \boldsymbol{w}\|_{q} .
\end{gathered}
$$

Proof of Proposition 3.1: We observe that from estimate (8) and the embedding $\mathbf{W}^{1, m}(\Omega) \subset \mathbf{L}^{3}(\Omega)$ we obtain

$$
\begin{aligned}
\frac{1}{\mu+\mu_{r}}\left\|P\{\overline{\boldsymbol{u}} \cdot \nabla \overline{\boldsymbol{u}}\}-2 \mu_{r} \operatorname{rot} \overline{\boldsymbol{w}}\right\|_{3} & \leq \frac{c_{1}}{\mu+\mu_{r}}\|A \overline{\boldsymbol{u}}\|_{3}^{2}+\frac{2 \mu_{r}}{\mu+\mu_{r}}\|\operatorname{rot} \overline{\boldsymbol{w}}\|_{3} \\
& \leq \frac{c_{1}}{\mu+\mu_{r}}\|A \overline{\boldsymbol{u}}\|_{3}^{2}+\frac{2 c_{2} \mu_{r}}{\mu+\mu_{r}}\|B \overline{\boldsymbol{w}}\|_{m} .
\end{aligned}
$$

On the other hand, from the inequality (9) and the embedding $\mathbf{W}^{1,3}(\Omega) \subset$ $\mathbf{L}^{m}(\Omega)$ we have

$$
\begin{aligned}
& \frac{1}{\chi}\left\|\overline{\boldsymbol{u}} \cdot \nabla \overline{\boldsymbol{w}}+4 \mu_{r} \overline{\boldsymbol{w}}-\gamma \nabla \operatorname{div} \overline{\boldsymbol{w}}-2 \mu_{r} \operatorname{rot} \overline{\boldsymbol{u}}\right\|_{m} \\
& \quad \leq \frac{c_{3}}{\chi}\|A \overline{\boldsymbol{u}}\|_{3}\|B \overline{\boldsymbol{w}}\|_{m}+\frac{4 \mu_{r}}{\chi}\|\overline{\boldsymbol{w}}\|_{m}+\frac{\gamma}{\chi}\|\nabla \operatorname{div} \overline{\boldsymbol{w}}\|_{m}+\frac{2 \mu_{r}}{\chi}\|\operatorname{rot} \overline{\boldsymbol{u}}\|_{m} \\
& \quad \leq \frac{c_{3}}{\chi}\|A \overline{\boldsymbol{u}}\|_{3}\|B \overline{\boldsymbol{w}}\|_{m}+\frac{4 c_{4} \mu_{r}}{\chi}\|B \overline{\boldsymbol{w}}\|_{m}+\frac{c_{5} \gamma}{\chi}\|B \overline{\boldsymbol{w}}\|_{m}+\frac{2 c_{6} \mu_{r}}{\chi}\|A \overline{\boldsymbol{u}}\|_{3} .
\end{aligned}
$$

Therefore, if $\|A \overline{\boldsymbol{u}}\|_{3}+\|B \overline{\boldsymbol{w}}\|_{m} \leq K_{0}$, we have

$$
\begin{aligned}
\left\|F[\overline{\boldsymbol{u}}, \overline{\boldsymbol{w}}]^{T}\right\|_{D\left(A_{3}\right) \times D\left(B_{m}\right)} & \leq \widetilde{C}\left(\|A \overline{\boldsymbol{u}}\|_{3}+\|B \overline{\boldsymbol{w}}\|_{m}\right)+\widetilde{C}_{1}\left(\|A \overline{\boldsymbol{u}}\|_{3}+\|B \overline{\boldsymbol{w}}\|_{m}\right)^{2} \\
& \leq \widetilde{C} K_{0}+\widetilde{C}_{1} K_{0}^{2},
\end{aligned}
$$

being $\widetilde{C}, \widetilde{C}_{1}$ given by $\widetilde{C}=\max \left(\frac{2 c_{2} \mu_{r}}{\mu+\mu_{r}}+\frac{4 c_{4} \mu_{r}}{\chi}+\frac{c_{5} \gamma}{\chi}, \frac{2 c_{6} \mu_{r}}{\chi}\right), \widetilde{C}_{1}=\max \left(\frac{c_{1}}{\mu+\mu_{r}}, \frac{c_{3}}{\chi}\right)$. Let $K_{0}=(1-\widetilde{C}) / \widetilde{C}_{1}$. Then, if $\mu$ and $\chi$ are large enough such that $\widetilde{C}<1$, we have $F\left(E_{K_{0}}\right) \subset E_{K_{0}}$ (that is, $E_{K_{0}}$ is an invariant set in $D\left(A_{3}\right) \times D\left(B_{m}\right)$ ).

Remark 3.1 Observe that in Theorem 2.2, the hypothesis $\mu$ and $\chi$ large enough, can be replaced by the hypothesis $\mu_{r}$ and $\gamma$ small enough such that $\widetilde{C}<1$. 
Using the arguments appearing in inequalities (10)-(11), we can infer that

$$
\begin{aligned}
\left\|F\left[\overline{\boldsymbol{u}}_{1}, \overline{\boldsymbol{w}}_{1}\right]^{T}-F\left[\overline{\boldsymbol{u}}_{2}, \overline{\boldsymbol{w}}_{2}\right]^{T}\right\|_{D\left(A_{3}\right) \times D\left(B_{m}\right)} \leq \\
\leq \widetilde{C}_{2}\left\{\left\|A\left(\overline{\boldsymbol{u}}_{1}-\overline{\boldsymbol{u}}_{2}\right)\right\|_{3}+\left\|B\left(\overline{\boldsymbol{w}}_{1}-\overline{\boldsymbol{w}}_{2}\right)\right\|_{m}\right\},
\end{aligned}
$$

for $\left(\boldsymbol{u}_{1}, \boldsymbol{w}_{1}\right),\left(\boldsymbol{u}_{2}, \boldsymbol{w}_{2}\right) \in E_{K_{0}}$, and a positive constant $\widetilde{C}_{2}=\widetilde{C}_{2}\left(\mu, \mu_{r}, \chi, \gamma, m, K_{0}\right)$. Indeed,

$$
\begin{aligned}
& \frac{1}{\mu+\mu_{r}}\left\|P\left(\overline{\boldsymbol{u}}_{1} \cdot \nabla \overline{\boldsymbol{u}}_{1}\right)-P\left(\overline{\boldsymbol{u}}_{2} \cdot \nabla \overline{\boldsymbol{u}}_{2}\right)-2 \mu_{r} \operatorname{rot}\left(\overline{\boldsymbol{w}}_{1}-\overline{\boldsymbol{w}}_{2}\right)\right\|_{3} \\
& \quad \leq \frac{c_{1}}{\mu+\mu_{r}}\left\|A\left(\overline{\boldsymbol{u}}_{1}-\overline{\boldsymbol{u}}_{2}\right)\right\|_{3}\left(\left\|A \overline{\boldsymbol{u}}_{1}\right\|_{3}+\left\|A \overline{\boldsymbol{u}}_{2}\right\|_{3}\right)+\frac{2 c_{2} \mu_{r}}{\mu+\mu_{r}}\left\|B\left(\overline{\boldsymbol{w}}_{1}-\overline{\boldsymbol{w}}_{2}\right)\right\|_{m} \\
& \quad \leq \frac{2 c_{1} K_{0}}{\mu+\mu_{r}}\left\|A\left(\overline{\boldsymbol{u}}_{1}-\overline{\boldsymbol{u}}_{2}\right)\right\|_{3}+\frac{2 c_{2} \mu_{r}}{\mu+\mu_{r}}\left\|B\left(\overline{\boldsymbol{w}}_{1}-\overline{\boldsymbol{w}}_{2}\right)\right\|_{m} ;
\end{aligned}
$$

and also,

$$
\begin{aligned}
& \frac{1}{\chi}\left\|\overline{\boldsymbol{u}}_{1} \cdot \nabla \overline{\boldsymbol{w}}_{1}-\overline{\boldsymbol{u}}_{2} \cdot \nabla \overline{\boldsymbol{w}}_{2}+4 \mu_{r}\left(\overline{\boldsymbol{w}}_{1}-\overline{\boldsymbol{w}}_{2}\right)-\gamma \nabla \operatorname{div}\left(\overline{\boldsymbol{w}}_{1}-\overline{\boldsymbol{w}}_{2}\right)-2 \mu_{r} \operatorname{rot}\left(\overline{\boldsymbol{u}}_{1}-\overline{\boldsymbol{u}}_{2}\right)\right\|_{m} \\
& \quad \leq \frac{c_{3}}{\chi}\left(\left\|A\left(\overline{\boldsymbol{u}}_{1}-\overline{\boldsymbol{u}}_{2}\right)\right\|_{3}\left\|B \overline{\boldsymbol{w}}_{1}\right\|_{m}\right)+\frac{c_{3}}{\chi}\left(\left\|A \overline{\boldsymbol{u}}_{2}\right\|_{3}\left\|B\left(\overline{\boldsymbol{w}}_{1}-\overline{\boldsymbol{w}}_{2}\right)\right\|_{m}\right) \\
& \quad+\frac{4 c_{4} \mu_{r}}{\chi}\left\|B\left(\overline{\boldsymbol{w}}_{1}-\overline{\boldsymbol{w}}_{2}\right)\right\|_{m}+\frac{c_{5} \gamma}{\chi}\left\|B\left(\overline{\boldsymbol{w}}_{1}-\overline{\boldsymbol{w}}_{2}\right)\right\|_{m}+\frac{2 c_{6} \mu_{r}}{\chi}\left\|A\left(\overline{\boldsymbol{u}}_{1}-\overline{\boldsymbol{u}}_{2}\right)\right\|_{3} \\
& \leq \frac{c_{3} K_{0}}{\chi}\left(\left\|A\left(\overline{\boldsymbol{u}}_{1}-\overline{\boldsymbol{u}}_{2}\right)\right\|_{3}+\left\|B\left(\overline{\boldsymbol{w}}_{1}-\overline{\boldsymbol{w}}_{2}\right)\right\|_{m}\right) \\
& \quad+\frac{4 c_{4} \mu_{r}}{\chi}\left\|B\left(\overline{\boldsymbol{w}}_{1}-\overline{\boldsymbol{w}}_{2}\right)\right\|_{m}+\frac{c_{5} \gamma}{\chi}\left\|B\left(\overline{\boldsymbol{w}}_{1}-\overline{\boldsymbol{w}}_{2}\right)\right\|_{m}+\frac{2 c_{6} \mu_{r}}{\chi}\left\|A\left(\overline{\boldsymbol{u}}_{1}-\overline{\boldsymbol{u}}_{2}\right)\right\|_{3} .
\end{aligned}
$$

So, we find (12) with $\widetilde{C}_{2}$ given by

$$
\widetilde{C}_{2}=\max \left(\frac{2 c_{1} K_{0}}{\mu+\mu_{r}}+\frac{c_{3} K_{0}}{\chi}+\frac{2 c_{6} \mu_{r}}{\chi}, \frac{2 c_{2} \mu_{r}}{\mu+\mu_{r}}+\frac{c_{3} K_{0}}{\chi}+\frac{4 c_{4} \mu_{r}}{\chi}+\frac{c_{5} \gamma}{\chi}\right) .
$$

We choose $\bar{\delta}(m)$ such that for $\mu, \chi \geq \bar{\delta}(m)$, we shall obtain $\widetilde{C}_{2}<1$ (and therefore $\widetilde{C}<1$ ). With this choice, we have that $F$ is contractive, and, hence, the existence of a stationary solution is proved.

\section{Analysis of the Linearized Operator}

In this section we will make an analysis of the linearized operator $L=L_{p, q}$ with respect to the stationary solution $(\overline{\boldsymbol{u}}, \overline{\boldsymbol{w}})$ given by Theorem 2.2 , being $L=L_{0}+L_{1}$, where:

$$
L_{0}\left[\begin{array}{c}
\boldsymbol{u} \\
\boldsymbol{w}
\end{array}\right]=\left[\begin{array}{c}
\left(\mu+\mu_{r}\right) A \boldsymbol{u} \\
\chi B \boldsymbol{w}
\end{array}\right]
$$




$$
L_{1}\left[\begin{array}{c}
\boldsymbol{u} \\
\boldsymbol{w}
\end{array}\right]=\left[\begin{array}{c}
P\{(\overline{\boldsymbol{u}} \cdot \nabla) \boldsymbol{u}+(\boldsymbol{u} \cdot \nabla) \overline{\boldsymbol{u}}\}-2 \mu_{r} \operatorname{rot} \boldsymbol{w} \\
(\overline{\boldsymbol{u}} \cdot \nabla) \boldsymbol{w}+(\boldsymbol{u} \cdot \nabla) \overline{\boldsymbol{w}}+4 \mu_{r} \boldsymbol{w}-\gamma \nabla \operatorname{div} \boldsymbol{w}-2 \mu_{r} \operatorname{rot} \boldsymbol{u}
\end{array}\right],
$$

and whose definition domain is $D\left(L_{p, q}\right)=D\left(A_{p}\right) \times D\left(B_{q}\right)$, for $p, q \in(1, \infty)$ suitably chosen.

The following lemma provides one estimate of the operator $L_{1}$.

Lemma 4.1 Suppose that $p, q \in(1, \infty)$ satisfy

$$
-\frac{1}{3} \leq \frac{1}{p}-\frac{1}{q} \leq \frac{1}{3}, \frac{1}{p}-\frac{1}{q} \leq 1-\frac{1}{m}, \frac{1}{q}>\frac{1}{m}-\frac{1}{3} .
$$

Then, there exists a constant $C_{p, q}(m)>0$ such that:

$$
\begin{aligned}
\left\|L_{1}[\boldsymbol{u}, \boldsymbol{w}]^{T}\right\|_{\{p, q\}} & \leq C_{p, q}(m)\left\{\left(\|A \overline{\boldsymbol{u}}\|_{3}+\|B \overline{\boldsymbol{w}}\|_{m}\right)\|A \boldsymbol{u}\|_{p}\right. \\
& \left.+\|A \overline{\boldsymbol{u}}\|_{3}\|B \boldsymbol{w}\|_{q}+\mu_{r}\|A \boldsymbol{u}\|_{p}+\left(\mu_{r}+\gamma\right)\|B \boldsymbol{w}\|_{q}\right\},
\end{aligned}
$$

for all $(\boldsymbol{u}, \boldsymbol{w}) \in D\left(A_{p}\right) \times D\left(B_{q}\right)$.

Proof: The proof is a consequence of Lemma 3.2 together with the following embeddings

$\mathbf{W}^{1, q} \subset \mathbf{L}^{p}(\Omega), \quad \mathbf{W}^{1, p} \subset \mathbf{L}^{q}(\Omega), \quad D\left(A_{p}\right) \subset \mathbf{L}^{q}(\Omega)$.

For $0<\omega<\pi / 2$, we put $\Sigma_{\omega}=\{\lambda \in \mathbb{C}:|\arg \lambda|<\pi-\omega\} \cup\{0\}$. Then we have:

Lemma 4.2 Let $p, q$ be as in Lemma 4.1. Then for each $\omega \in(0, \pi / 2)$, there exists a constant $M_{p, q}(m, \omega)$ such that

$$
\left\|L_{1}\left(\lambda+L_{0}\right)^{-1}[\boldsymbol{u}, \boldsymbol{w}]^{T}\right\|_{\{p, q\}} \leq K_{p, q}\left\|[\boldsymbol{u}, \boldsymbol{w}]^{T}\right\|_{\{p, q\}},
$$

for all $\lambda \in \Sigma_{\omega}$ and $(\boldsymbol{u}, \boldsymbol{w}) \in \mathbf{L}_{\sigma}^{p}(\Omega) \times \mathbf{L}^{q}(\Omega)$, where

$$
K_{p, q} \equiv M_{p, q}\left\{\left(\mu_{r}+\gamma\right)+\left(\max \left\{\frac{1}{\mu+\mu_{r}}, \frac{1}{\chi}\right\}\right) K_{0}\right\},
$$

with $K_{0}$ the constant of Theorem 2.2.

Proof: It is well known (see Giga [8]) that for each $\omega \in(0, \pi / 2)$,

$$
\Sigma_{\omega} \subset \rho\left(-A_{p}\right) \cap \rho\left(-B_{q}\right),
$$

with

$$
\begin{aligned}
& K_{p}(\omega)=\sup _{\lambda \in \Sigma_{\omega}}(1+|\lambda|)\left\|(\lambda+A)^{-1}\right\|_{p \rightarrow p}<\infty, \\
& \hat{K}_{q}(\omega)=\sup _{\lambda \in \Sigma_{\omega}}(1+|\lambda|)\left\|(\lambda+B)^{-1}\right\|_{q \rightarrow q}<\infty .
\end{aligned}
$$


It remains to estimate $\left\|A\left(\lambda+\left(\mu+\mu_{r}\right) A\right)^{-1} \boldsymbol{u}\right\|_{p}$ and $\left\|B(\lambda+\chi B)^{-1} \boldsymbol{w}\right\|_{q}$. Observe that $A\left(\lambda+\left(\mu+\mu_{r}\right) A\right)^{-1}=\frac{1}{\mu+\mu_{r}} I-\frac{\lambda}{\mu+\mu_{r}}\left(\lambda+\left(\mu+\mu_{r}\right) A\right)^{-1}$, and therefore:

$\left\|A\left(\lambda+\left(\mu+\mu_{r}\right) A\right)^{-1} \boldsymbol{u}\right\|_{p} \leq \frac{1}{\mu+\mu_{r}}\|\boldsymbol{u}\|_{p}+\frac{|\lambda|}{\mu+\mu_{r}}\left\|\left(\lambda+\left(\mu+\mu_{r}\right) A\right)^{-1}\right\|_{p \rightarrow p}\|\boldsymbol{u}\|_{p}$.

Now, rewriting $\left(\lambda+\left(\mu+\mu_{r}\right) A\right)^{-1}=\frac{1}{\mu+\mu_{r}}\left(\frac{\lambda}{\mu+\mu_{r}}+A\right)^{-1}$, we have:

$\frac{|\lambda|}{\mu+\mu_{r}}\left\|\left(\lambda+\left(\mu+\mu_{r}\right) A\right)^{-1}\right\|_{p \rightarrow p}=\left(\frac{|\lambda|}{\mu+\mu_{r}}\right) \frac{1}{\mu+\mu_{r}}\left\|\left(\frac{\lambda}{\mu+\mu_{r}}+A\right)^{-1}\right\|_{p \rightarrow p}$.

If we call $\widetilde{\lambda}=\frac{\lambda}{\mu+\mu_{r}}$, we have:

$$
\frac{1}{\mu+\mu_{r}}|\widetilde{\lambda}|\left\|(\widetilde{\lambda}+A)^{-1}\right\|_{p \rightarrow p} \leq \frac{1}{\mu+\mu_{r}} K_{p}(\omega) .
$$

Estimates for $\left\|B(\lambda+\chi B)^{-1}\right\|_{q}$ are similar.

Remark 4.1 Observe that if $\lambda \in \Sigma_{\omega}$, then $\tilde{\lambda}=\frac{\lambda}{\mu+\mu_{r}} \in \Sigma_{\omega}$, because of $\arg \lambda=\arg \tilde{\lambda}$.

We recall that $\rho(\cdot)$ is the standard notation for the set resolvent and that $\|\cdot\|_{p \rightarrow q}$ denotes the norm of the bounded linear operator defined from $\mathbf{L}^{p}(\Omega)$ to $\mathbf{L}^{q}(\Omega)$. Taking into account that:

$$
\left(\lambda+L_{0}\right)^{-1}\left[\begin{array}{c}
\boldsymbol{u} \\
\boldsymbol{w}
\end{array}\right]=\left[\begin{array}{c}
\left(\lambda+\left(\mu+\mu_{r}\right) A\right)^{-1} \boldsymbol{u} \\
(\lambda+\chi B)^{-1} \boldsymbol{w}
\end{array}\right]
$$

by Lemma 4.1 and estimates (16)-(17) for $\widetilde{\lambda}$, we obtain:

$$
\begin{aligned}
& \left\|L_{1}\left(\lambda+L_{0}\right)^{-1}[\boldsymbol{u}, \boldsymbol{w}]^{T}\right\|_{\{p, q\}} \\
& \quad \leq C_{p, q}\left\{\left(\|A \overline{\boldsymbol{u}}\|_{3}+\|B \overline{\boldsymbol{w}}\|_{m}+\mu_{r}\right)\left(1+K_{p}(\omega)\right) \frac{1}{\left(\mu+\mu_{r}\right)}\|\boldsymbol{u}\|_{p}\right. \\
& \left.\quad+\left(\|A \overline{\boldsymbol{u}}\|_{3}+\mu_{r}+\gamma\right)\left(1+\hat{K}_{q}(\omega)\right) \frac{1}{\chi}\|\boldsymbol{w}\|_{q}\right\} .
\end{aligned}
$$

Since $(\overline{\boldsymbol{u}}, \overline{\boldsymbol{w}})$ belongs to the class $\left(m, K_{0}\right)$, we have $\|A \overline{\boldsymbol{u}}\|_{3}+\|B \overline{\boldsymbol{w}}\|_{m} \leq K_{0}$ and thus

$$
\begin{aligned}
\left\|L_{1}\left(\lambda+L_{0}\right)^{-1}[\boldsymbol{u}, \boldsymbol{w}]^{T}\right\|_{\{p, q\}} & \leq C_{p, q}\left\{\left(\mu_{r}+K_{0}\right)\left(1+K_{p}(\omega)\right) \frac{1}{\left(\mu+\mu_{r}\right)}\|\boldsymbol{u}\|_{p}\right. \\
& \left.+\left(\mu_{r}+\gamma+K_{0}\right)\left(1+\hat{K}_{q}(\omega)\right) \frac{1}{\chi}\|\boldsymbol{w}\|_{q}\right\} .
\end{aligned}
$$


Therefore, taking $M_{p, q}(m, \omega)=C_{p, q}\left(1+\hat{K}_{q}(\omega)+K_{p}(\omega)\right)$ we obtain (15).

Next, we will establish a lemma that will be fundamental in the calculus of the estimates $\left(\mathbf{L}^{p} \times \mathbf{L}^{q}\right)-\left(\mathbf{L}^{r} \times \mathbf{L}^{s}\right)$ of the resolvent of the operator $-L$.

Lemma 4.3 Let $p, q$ be as in Lemma 4.1. For each $\omega \in(0, \pi / 2)$ let

$$
\delta_{p, q}=\delta_{p, q}(m, \omega) \equiv \min \left\{\frac{1}{\left(1+K_{0}\right) M_{p, q}(m, \omega)}, \frac{1}{\bar{\delta}}\right\},
$$

being $\bar{\delta}, K_{0}$ and $M_{p, q}(m, \omega)$ the constants of Theorem 2.2 and Lemma 4.2. If

$$
\gamma+\mu_{r}<\delta_{p, q}, \quad \max \left\{\frac{1}{\mu+\mu_{r}}, \frac{1}{\chi}\right\}<\delta_{p, q},
$$

then $\Sigma_{\omega} \subset \rho\left(-L_{p, q}\right)$. Therefore, for each $j=0,1$, if $r$, s satisfy

$$
0 \leq \beta \equiv \frac{3}{2}\left(\frac{1}{p}-\frac{1}{r}\right) \leq 1-\frac{j}{2}, \quad 0 \leq \xi \equiv \frac{3}{2}\left(\frac{1}{q}-\frac{1}{s}\right) \leq 1-\frac{j}{2},
$$

then, there exists a positive constant $C=C\left(p, q, r, s, m, \omega, \mu, \mu_{r}, \chi, \gamma\right)$ such that: for all $\lambda \in \Sigma_{\omega}$ and $(\boldsymbol{u}, \boldsymbol{w}) \in \mathbf{L}_{\sigma}^{p}(\Omega) \times \mathbf{L}^{q}(\Omega)$

$$
\begin{aligned}
& \left\|\nabla^{j}(\lambda+L)^{-1}[\boldsymbol{u}, \boldsymbol{w}]^{T}\right\|_{\{r, s\}} \\
& \quad \leq C\left\{(1+|\lambda|)^{\beta+j / 2-1}+(1+|\lambda|)^{\xi+j / 2-1}\right\}\left\|[\boldsymbol{u}, \boldsymbol{w}]^{T}\right\|_{\{p, q\}} .
\end{aligned}
$$

Proof: From (18)-(19), we have:

$$
K_{p, q} \equiv M_{p, q}\left\{\left(\mu_{r}+\gamma\right)+\left(\max \left\{\frac{1}{\mu+\mu_{r}}, \frac{1}{\chi}\right\}\right) K_{0}\right\}<1 .
$$

Therefore, using estimate (15) appearing in Lemma 4.2, we know that:

$$
\left\|L_{1}\left(\lambda+L_{0}\right)^{-1}\right\|_{\{p, q\} \rightarrow\{p, q\}}<1
$$

and thus we deduce that for all $\lambda \in \Sigma_{\omega}$, there exists an inverse operator $[I+$ $\left.L_{1}\left(\lambda+L_{0}\right)^{-1}\right]^{-1}=\sum_{n=0}^{\infty}\left\{-L_{1}\left(\lambda+L_{0}\right)^{-1}\right\}^{n}$, on the space $\mathbf{L}_{\sigma}^{p}(\Omega) \times \mathbf{L}^{q}(\Omega)$, satisfying

$$
\left\|\left[I+L_{1}\left(\lambda+L_{0}\right)^{-1}\right]^{-1}\right\|_{\{p, q\} \rightarrow\{p, q\}} \leq \frac{1}{1-K_{p, q}} .
$$

Therefore,

$$
(\lambda+L)^{-1}=\left(\lambda+L_{0}\right)^{-1}\left[I+L_{1}\left(\lambda+L_{0}\right)^{-1}\right]^{-1}, \lambda \in \Sigma_{\omega},
$$

and it exists as a bounded linear operator on $\mathbf{L}_{\sigma}^{p}(\Omega) \times \mathbf{L}^{q}(\Omega)$. So, we obtain that $\Sigma_{\omega} \subset \rho\left(-L_{p, q}\right)$. 
In the following, we show estimate (21) for $j=0,1$. In a first time, we observe that from conditions (20), the embeddings $D\left(A_{p}^{\beta+j / 2}\right) \subset W^{j, r}(\Omega)$ and $D\left(B_{q}^{\xi+j / 2}\right) \subset W^{j, s}(\Omega)$ are true. These relations imply the following estimate

$$
\begin{aligned}
\| & \nabla^{j}\left(\lambda+L_{0}\right)^{-1}[\boldsymbol{u}, \boldsymbol{w}]^{T} \|_{\{r, s\}} \\
& =\left\|\nabla^{j}\left(\lambda+\left(\mu+\mu_{r}\right) A\right)^{-1} \boldsymbol{u}\right\|_{r}+\left\|\nabla^{j}(\lambda+\chi B)^{-1} \boldsymbol{w}\right\|_{s} \\
& \leq C\left\|A^{\beta+j / 2}\left(\lambda+\left(\mu+\mu_{r}\right) A\right)^{-1} \boldsymbol{u}\right\|_{p}+C\left\|B^{\xi+j / 2}(\lambda+\chi B)^{-1} \boldsymbol{w}\right\|_{q} \\
& \leq C\left\|A\left(\lambda+\left(\mu+\mu_{r}\right) A\right)^{-1} \boldsymbol{u}\right\|_{p}^{\beta+j / 2}\left\|\left(\lambda+\left(\mu+\mu_{r}\right) A\right)^{-1} \boldsymbol{u}\right\|_{p}^{1-\beta-j / 2} \\
& +C\left\|B(\lambda+\chi B)^{-1} \boldsymbol{w}\right\|_{q}^{\xi+j / 2}\left\|(\lambda+\chi B)^{-1} \boldsymbol{w}\right\|_{q}^{1-\xi-j / 2} \\
& \leq \frac{C}{\mu+\mu_{r}}\left(1+K_{p}(\omega)\right)\left(1+\frac{1}{\mu+\mu_{r}}|\lambda|\right)^{\beta+j / 2-1}\|\boldsymbol{u}\|_{p} \\
& +\frac{C}{\chi}\left(1+\hat{K}_{q}(\omega)\right)\left(1+\frac{1}{\chi}|\lambda|\right)^{\xi+j / 2-1}\|\boldsymbol{w}\|_{q},
\end{aligned}
$$

for all $\lambda \in \Sigma_{\omega}$ and $(\boldsymbol{u}, \boldsymbol{w}) \in \mathbf{L}_{\sigma}^{p}(\Omega) \times \mathbf{L}^{q}(\Omega)$. Observe that we have used Proposition 2.3.3 of Tanabe [29], that reads:

$$
\left\|A^{\alpha} \boldsymbol{u}\right\| \leq C_{\alpha, \beta}\left\|A^{\beta} \boldsymbol{u}\right\|^{\alpha / \beta}\|\boldsymbol{u}\|^{1-\alpha / \beta} \quad \text { for } 0 \leq \alpha \leq \beta \leq 1 \text { and } \boldsymbol{u} \in D\left(A^{\beta}\right) .
$$

Consequently, from (22)-(23) and (24) we obtain (21).

Below, we fix $\omega \in(0, \pi / 2)$ but arbitrary; therefore, the constant $\delta_{p, q}$ of Lemma 4.3 depends only on $p, q, m$. Lemma 4.3 is useful in order to show the generation of analytic semigroup and also of $\left(\mathbf{L}^{p} \times \mathbf{L}^{q}\right)-\left(\mathbf{L}^{r} \times \mathbf{L}^{s}\right)$ estimates.

Proposition 4.4 Let $p, q$ be as in Lemma 4.1 and assume that $\mu, \mu_{r}, \chi, \gamma$ satisfy (19). Then, the operator $-L$ generates a bounded analytic semigroup $\left\{e^{-t L}\right\}_{t \geq 0}$ of class $C_{0}$ on the space $\mathbf{L}_{\sigma}^{p} \times \mathbf{L}^{q}(\Omega)$. Moreover, for $j=0,1$ and $r, s$ satisfying (20), there exists a positive constant $C=C\left(p, q, r, s, m, \mu, \mu_{r}, \chi, \gamma\right)$ such that: for all $t>0$ and $(\boldsymbol{u}, \boldsymbol{w}) \in \mathbf{L}_{\sigma}^{p}(\Omega) \times \mathbf{L}^{q}(\Omega)$

$$
\begin{aligned}
& \left\|\nabla^{j} e^{-t L}[\boldsymbol{u}, \boldsymbol{w}]^{T}\right\|_{\{r, s\}} \\
& \quad \leq C\left\{t^{-(3 / 2)(1 / p-1 / r)-j / 2}+t^{-(3 / 2)(1 / q-1 / s)-j / 2}\right\}\left\|[\boldsymbol{u}, \boldsymbol{w}]^{T}\right\|_{\{p, q\}} .
\end{aligned}
$$

Proof: Using estimate $(21)(j=0)$ for $\{r, s\}=\{p, q\}$ from the classical results of analytic semigroup, we obtain the first part of the proof (see, for example, Tanabe [29] or Pazy [24] for a version of this result). In order to obtain the inequality $(25)_{j}$, as usual, we compute the Dunford integral

$$
\nabla^{j} e^{-t L}=\frac{1}{2 \pi i} \int_{\Gamma} \nabla^{j}(\lambda+L)^{-1} e^{t \lambda} d \lambda, t>0,
$$

using inequality (21). Here, when $\beta+j / 2$ and $\xi+j / 2$ are positive, the resolvent is integrated from $\infty e^{-i \varphi}$ to $\infty e^{i \varphi}$ along the path $\Gamma: \lambda=|\lambda| e^{ \pm i \varphi}$ for a $\varphi \in$ 
$(\pi / 2, \pi-\omega)$ fix but arbitrary. When $\beta+j / 2=0$, that is, $r=p$ and $j=0$, we divide the Dunford integral in two parts and we replace $\Gamma$ by $\bar{\Gamma}=\Gamma_{t} \cup \Gamma_{1}$ where $\Gamma_{t}: \lambda=|\lambda| e^{ \pm i \varphi},(|\lambda| \geq 1 / t)$ and $\Gamma_{1}:(1 / t) e^{i \arg \lambda},-\varphi \leq \arg \lambda \leq \varphi$. Thus,

$$
\left\|e^{-t L}\right\|_{\{p, q\} \rightarrow\{p, s\}} \leq \frac{c}{2 \pi} \int_{\bar{\Gamma}}|\lambda|^{-1} e^{t \operatorname{Re} \lambda}|d \lambda|+\frac{c}{2 \pi} \int_{\Gamma}|\lambda|^{-1+\xi} e^{t \operatorname{Re} \lambda}|d \lambda| .
$$

The case $\xi+j / 2=0$ is done analogously. An elementary computation implies the desired estimates $(25)_{j}$.

Remark 4.2 We observe that there exists a positive number $\zeta$ satisfying

$$
\operatorname{Re} \sigma\left(L_{p, q}\right)>\zeta
$$

where $\sigma(\cdot)$ represents the spectrum of the operator $L_{p, q}$. This is equivalent to that $\sigma\left(L_{p, q}\right) \subset\{\lambda \in \mathbb{C}: \operatorname{Re} \lambda>\zeta\}$. This property is useful to derive the exponential decay of $e^{-t L}$, when $t \rightarrow \infty$.

Proposition 4.5 Let $p, q$ be as in Lemma 4.1 and assume that condition (19) is satisfied. If $\zeta>0$ satisfies (26), then there exists a positive constant $C_{\zeta}=$ $C_{\zeta}\left(p, q, \mu, \mu_{r}, \chi, \gamma\right)$ such that: for all $t \geq 0$ and $(\boldsymbol{u}, \boldsymbol{w}) \in \mathbf{L}_{\sigma}^{p}(\Omega) \times \mathbf{L}^{q}(\Omega)$

$$
\left\|e^{-t L}[\boldsymbol{u}, \boldsymbol{w}]^{T}\right\|_{\{p, q\}} \leq C_{\zeta} e^{-\zeta t}\left\|[\boldsymbol{u}, \boldsymbol{w}]^{T}\right\|_{\{p, q\}} .
$$

Proposition 4.6 Let $p, q$ be as in Lemma 4.1 and assume condition (19). If $\zeta>0$ satisfies (26), then for $j=0,1$ and $\{r, s\}$ satisfying (20) there exists a positive constant $C_{\zeta}=C_{\zeta}\left(r, s, p, q, m, \mu, \mu_{r}, \chi, \gamma\right)$ such that: for all $t>0$ and $(\boldsymbol{u}, \boldsymbol{v}) \in \mathbf{L}_{\sigma}^{p}(\Omega) \times \mathbf{L}^{q}(\Omega)$

$$
\begin{aligned}
& \left\|\nabla^{j} e^{-t L}[\boldsymbol{u}, \boldsymbol{w}]^{T}\right\|_{\{r, s\}} \\
& \quad \leq C_{\zeta}\left\{t^{-(3 / 2)(1 / p-1 / r)-j / 2}+t^{-(3 / 2)(1 / q-1 / s)-j / 2}\right\} e^{-\zeta t}\left\|[\boldsymbol{u}, \boldsymbol{w}]^{T}\right\|_{\{p, q\}} .
\end{aligned}
$$

Lemma 4.7 For any $\zeta>0$ satisfying (26) there exists $\zeta^{*}>\zeta$ such that $\zeta^{*}$ also satisfies (26), that is, $\operatorname{Re} \sigma\left(L_{p, q}\right)>\zeta^{*}$.

Proof of Lemma 4.7: The proof is an easy consequence from the fact that $\sigma\left(L_{p, q}\right)$ is a closed set.

Proof of Proposition 4.5: By using Lemma 4.7 and recalling that $\Sigma_{\omega} \subset$ $\rho\left(-L_{p, q}\right)$ we have that there exists a number $\zeta^{*}>\zeta$ such that

$$
\Theta_{\omega}^{\zeta^{*}} \equiv \Sigma_{\omega} \cup\left\{\lambda \in \mathbb{C}: \operatorname{Re} \lambda \geq-\zeta^{*}\right\} \subset \rho\left(-L_{p, q}\right) .
$$

We can take $\varphi=\varphi\left(\zeta^{*}\right) \in(\pi / 2, \pi)$ such that $\Gamma \equiv\{\lambda \in \mathbb{C}: \lambda=-\zeta+\mid \lambda+$ $\left.\zeta \mid e^{ \pm i \varphi}\right\} \subset \Theta_{\omega}^{\zeta^{*}}$. From (21) with $j=0$ we find that $\left\|(\lambda+L)^{-1}\right\|_{\{p, q\} \rightarrow\{p, q\}} \leq C_{\zeta^{*}}$, $\lambda \in \Theta_{\omega}^{\zeta^{*}}$, where $C_{\zeta^{*}}=C_{\zeta^{*}}\left(p, q, \mu, \mu_{r}, m, \chi, \gamma\right)$. Therefore,

$$
\begin{aligned}
& \left\|e^{-t L}[\boldsymbol{u}, \boldsymbol{w}]^{T}\right\|_{\{p, q\}}=\frac{1}{2 \pi}\left\|\int_{\Gamma}(\lambda+L)^{-1} e^{t \lambda} d \lambda[\boldsymbol{u}, \boldsymbol{w}]^{T}\right\|_{\{p, q\}} \\
& \quad \leq \frac{C_{\zeta^{*}} e^{-\zeta t}}{\pi} \int_{0}^{\infty} e^{t \eta \cos \varphi} d \eta \cdot\left\|[\boldsymbol{u}, \boldsymbol{w}]^{T}\right\|_{\{p, q\}}=\frac{-C_{\zeta^{*}} e^{-\zeta t}}{\pi t \cos \varphi}\left\|[\boldsymbol{u}, \boldsymbol{w}]^{T}\right\|_{\{p, q\}},
\end{aligned}
$$


for $t>0$. If $t>1$, estimate (27) is easily obtained. If $t<1$, the estimates of the semigroup given by Proposition 4.4 imply (27).

Proof of Proposition 4.6: Given $\zeta>0$, by Lemma 4.7, we choose $\tau=\tau(\zeta)>$ 0 small enough such that $\zeta /(1-\tau)$ also satisfies (26). From Proposition 4.5,

$$
\left\|e^{-t L}[\boldsymbol{u}, \boldsymbol{w}]^{T}\right\|_{\{p, q\}} \leq C_{\zeta /(1-\tau)} e^{-(\zeta /(1-\tau)) t}\left\|[\boldsymbol{u}, \boldsymbol{w}]^{T}\right\|_{\{p, q\}}, \quad t \geq 0 .
$$

The last inequality together with Proposition 4.4 imply

$$
\begin{aligned}
& \left\|\nabla^{j} e^{-t L}\left[\begin{array}{c}
\boldsymbol{u} \\
\boldsymbol{w}
\end{array}\right]\right\|_{\{r, s\}}=\left\|\nabla^{j} e^{-\tau t L} e^{-(1-\tau) t L}\left[\begin{array}{c}
\boldsymbol{u} \\
\boldsymbol{w}
\end{array}\right]\right\|_{\{r, s\}} \\
& \leq C\left\{(\tau t)^{-(3 / 2)(1 / p-1 / r)-j / 2}+(\tau t)^{-(3 / 2)(1 / q-1 / s)-j / 2}\right\}\left\|e^{-(1-\tau) t L}\left[\begin{array}{c}
\boldsymbol{u} \\
\boldsymbol{w}
\end{array}\right]\right\|_{\{p, q\}} \\
& \leq C_{\zeta}\left\{t^{-(3 / 2)(1 / p-1 / r)-j / 2}+t^{-(3 / 2)(1 / q-1 / s)-j / 2}\right\} e^{-\zeta t}\left\|\left[\begin{array}{c}
\boldsymbol{u} \\
\boldsymbol{w}
\end{array}\right]\right\|_{\{p, q\}}
\end{aligned}
$$

This inequality proves (28).

\section{Proof of Theorem 2.4}

In this section, we study the global existence and the exponential decay of solutions for the micropolar fluid system. Let $(\overline{\boldsymbol{u}}, \overline{\boldsymbol{w}})$ be the stationary solution of class $\left(m, K_{0}\right)$ given by Theorem 2.2. Consequently, if we write $(\widetilde{\boldsymbol{u}}, \widetilde{\boldsymbol{w}})=$ $(\boldsymbol{u}-\overline{\boldsymbol{u}}, \boldsymbol{w}-\overline{\boldsymbol{w}})$, we have that $(\overline{\boldsymbol{u}}, \overline{\boldsymbol{w}})$ satisfies the system (5). This system can be rewritten as

$$
\left\{\begin{aligned}
& \frac{d}{d t}\left[\begin{array}{c}
\widetilde{\boldsymbol{u}} \\
\widetilde{\boldsymbol{w}}
\end{array}\right]+L\left[\begin{array}{c}
\widetilde{\boldsymbol{u}} \\
\widetilde{\boldsymbol{w}}
\end{array}\right]+\left[\begin{array}{c}
P(\widetilde{\boldsymbol{u}} \cdot \nabla \widetilde{\boldsymbol{u}}) \\
\widetilde{\boldsymbol{u}} \cdot \nabla \widetilde{\boldsymbol{w}}
\end{array}\right]=\left[\begin{array}{l}
0 \\
0
\end{array}\right], t>0 \\
& \widetilde{\boldsymbol{u}}(0)=\boldsymbol{u}_{0}-\overline{\boldsymbol{u}}, \quad \widetilde{\boldsymbol{w}}(0)=\boldsymbol{w}_{0}-\overline{\boldsymbol{w}}
\end{aligned}\right.
$$

in adequate spaces $\mathbf{L}_{\sigma}^{p}(\Omega) \times \mathbf{L}^{q}(\Omega)$, where $L$ is the linearized operator studied in the above section. Since $-L$ generates a bounded analytic semigroup, the integral formulation associated to (29), i. e., the mild formulation, is given by

$$
\left[\begin{array}{c}
\widetilde{\boldsymbol{u}}(t) \\
\widetilde{\boldsymbol{w}}(t)
\end{array}\right]=e^{-t L}\left[\begin{array}{c}
\boldsymbol{u}_{0}-\overline{\boldsymbol{u}} \\
\boldsymbol{w}_{0}-\overline{\boldsymbol{w}}
\end{array}\right]-\int_{0}^{t} e^{-(t-s) L}\left[\begin{array}{c}
P(\widetilde{\boldsymbol{u}} \cdot \nabla \widetilde{\boldsymbol{u}}) \\
\widetilde{\boldsymbol{u}} \cdot \nabla \widetilde{\boldsymbol{w}}
\end{array}\right](s) d s .
$$

To obtain the decay estimates, firstly we will prove a result on global existence.

Proposition 5.1 Let $m$ and $r_{0}$ satisfying

$$
\frac{3}{2} \leq m<3, \quad 3<r_{0}<\infty .
$$

Assume the initial data $\left(\boldsymbol{u}_{0}-\overline{\boldsymbol{u}}, \boldsymbol{w}_{0}-\overline{\boldsymbol{w}}\right) \in \mathbf{L}_{\sigma}^{3}(\Omega) \times \mathbf{L}^{m}(\Omega)$ and consider the constant $\delta_{p, q}(m)$ given by (18). Let $\delta^{\prime}=\delta^{\prime}\left(m, r_{0}\right) \equiv \min \left\{\delta_{3, m}, \delta_{3 r_{0} /\left(3+r_{0}\right), m r_{0} /\left(m+r_{0}\right)}\right\}$. 
If $\gamma+\mu_{r}<\delta^{\prime}$ and $\max \left\{\frac{1}{\mu+\mu_{r}}, \frac{1}{\chi}\right\}<\delta^{\prime}$, then there exists a positive constant $\epsilon=\epsilon\left(m, r_{0}, \mu, \mu_{r}, \chi, \gamma\right)$ such that if

$$
\left\|\boldsymbol{u}_{0}-\overline{\boldsymbol{u}}\right\|_{3}+\left\|\boldsymbol{w}_{0}-\overline{\boldsymbol{w}}\right\|_{m}<\epsilon,
$$

the system (29) has a unique strong solution $(\widetilde{\boldsymbol{u}}, \widetilde{\boldsymbol{w}}) \in \mathbf{L}_{\sigma}^{3}(\Omega) \times \mathbf{L}^{m}(\Omega)$ defined on the interval $[0, \infty)$. Moreover, if $\zeta>0$ satisfies (26) for $\{p, q\}=\left\{3 r_{0} /(3+\right.$ $\left.\left.r_{0}\right), m r_{0} /\left(m+r_{0}\right)\right\}$ and $\{3, m\}$, then the following estimates hold

$$
\begin{array}{r}
\|\widetilde{\boldsymbol{u}}(t)\|_{r} \leq C_{\zeta} t^{-(3 / 2)(1 / 3-1 / r)} e^{-\zeta t}\left(\left\|\boldsymbol{u}_{0}-\overline{\boldsymbol{u}}\right\|_{3}+\left\|\boldsymbol{w}_{0}-\overline{\boldsymbol{w}}\right\|_{m}\right), \\
\|\widetilde{\boldsymbol{w}}(t)\|_{s} \leq \widetilde{C}_{\zeta} t^{-(3 / 2)(1 / m-1 / s)} e^{-\zeta t}\left(\left\|\boldsymbol{u}_{0}-\overline{\boldsymbol{u}}\right\|_{3}+\left\|\boldsymbol{w}_{0}-\overline{\boldsymbol{w}}\right\|_{m}\right), \\
\|\nabla \widetilde{\boldsymbol{u}}(t)\|_{r_{1}} \leq C_{\zeta}^{\prime} t^{-(3 / 2)\left(1 / 3-1 / r_{1}\right)-1 / 2} e^{-\zeta t}\left(\left\|\boldsymbol{u}_{0}-\overline{\boldsymbol{u}}\right\|_{3}+\left\|\boldsymbol{w}_{0}-\overline{\boldsymbol{w}}\right\|_{m}\right), \\
\|\nabla \widetilde{\boldsymbol{w}}(t)\|_{s_{1}} \leq \widetilde{C}_{\zeta}^{\prime} t^{-(3 / 2)\left(1 / m-1 / s_{1}\right)-1 / 2} e^{-\zeta t}\left(\left\|\boldsymbol{u}_{0}-\overline{\boldsymbol{u}}\right\|_{3}+\left\|\boldsymbol{w}_{0}-\overline{\boldsymbol{w}}\right\|_{m}\right),
\end{array}
$$

for $3 \leq r<\infty, m \leq s<3 m /(3-m), 3 \leq r_{1}<r_{0}, m \leq s_{1}<(1 / m+$ $\left.1 / r_{0}-1 / 3\right)^{-1}$, and for all $t>0$, being $C_{\zeta}=C_{\zeta}\left(r, \mu, \mu_{r}, \gamma, \chi, m, r_{0}\right), C_{\zeta}^{\prime}=$ $C_{\zeta}\left(r_{1}, \mu, \mu_{r}, \gamma, \chi, m, r_{0}\right), \widetilde{C}_{\zeta}=\widetilde{C}_{\zeta}\left(s, \mu, \mu_{r}, \chi, \gamma, m, r_{0}\right), \widetilde{C}_{\zeta}^{\prime}=\widetilde{C}_{\zeta}^{\prime}\left(s_{1}, \mu, \mu_{r}, \chi, \gamma, m, r_{0}\right)$.

Remark 5.1 The constant $\delta^{\prime}$ in Proposition 5.1 is well defined since $\{3, m\}$ and $\left\{3 r_{0} /\left(3+r_{0}\right), m r_{0} /\left(m+r_{0}\right)\right\}$ satisfy (13).

Remark 5.2 We observe that in Proposition 5.1, $m \in[3 / 2,3)$. For $m \in[3, \infty)$, we consider $3 / 2<r_{0}<\infty$ in the hypothesis of Proposition 5.1 and we obtain the same result. We will focus the analysis on the case $m \in[3 / 2,3)$ because in this case the difficulty to derive a decay estimate of microrotation velocity $\|\widetilde{\boldsymbol{w}}\|_{\infty}$, seems greater.

Remark 5.3 We observe that if we take $\overline{\boldsymbol{u}}=\overline{\boldsymbol{w}}=0$ and $m=3$, the result of Proposition 5.1 (together with Remark 5.2) we obtain a result of existence of solutions with the same regularity for $(\boldsymbol{u}, \boldsymbol{w})$ than in Yamaguchi's work [31].

To prove this proposition, we precise of the following two lemmas.

Lemma 5.2 Let $m$ be as in Proposition 5.1 and $\zeta>0$ satisfying (26) for $\{p, q\}=\{3, m\}$. Assume also that

$$
\gamma+\mu_{r}<\delta_{3, m}, \quad \max \left\{\frac{1}{\mu+\mu_{r}}, \frac{1}{\chi}\right\}<\delta_{3, m}
$$

If $\{r, s\}$ satisfy

$$
\begin{gathered}
3 \leq r<\infty, \\
\frac{1}{3}-\frac{1}{r}=\frac{1}{m}-\frac{1}{s},
\end{gathered}
$$

then: for all $t>0, j=0,1$, and $(\boldsymbol{u}, \boldsymbol{w}) \in \mathbf{L}_{\sigma}^{3}(\Omega) \times \mathbf{L}^{m}(\Omega)$,

$$
\left\|\nabla^{j} e^{-t L}[\boldsymbol{u}, \boldsymbol{w}]^{T}\right\|_{\{r, s\}} \leq C_{\zeta} t^{-(3 / 2)(1 / 3-1 / r)-j / 2} e^{-\zeta t}\left\|[\boldsymbol{u}, \boldsymbol{w}]^{T}\right\|_{\{3, m\}},
$$

being $C_{\zeta}=C_{\zeta}\left(r, m, \mu, \mu_{r}, \chi, \gamma\right)$. 
Proof: By condition (31) we can see that $\{3, m\}$ satisfies (13). Moreover, the condition (38) together with $0 \leq \frac{3}{2}\left(\frac{1}{3}-\frac{1}{r}\right) \leq \frac{1}{2} \leq 1-\frac{j}{2}, j=0,1$, implies that $\{r, s\}$ satisfies (20). Consequently, inequality (39) is a consequence of Proposition 4.6, because of hypothesis (36) is the correspondent hypothesis (19) of Proposition 4.6.

Lemma 5.3 Let $m, r_{0}$ be as in Proposition 5.1 and $\zeta>0$ satisfying (26) for $\{p, q\}=\left\{3 r_{0} /\left(3+r_{0}\right), m r_{0} /\left(m+r_{0}\right)\right\}$. Assume also that

$$
\gamma+\mu_{r}<\delta_{3 r_{0} /\left(3+r_{0}\right), m r_{0} /\left(m+r_{0}\right)}, \quad \max \left\{\frac{1}{\mu+\mu_{r}}, \frac{1}{\chi}\right\}<\delta_{3 r_{0} /\left(3+r_{0}\right), m r_{0} /\left(m+r_{0}\right)} .
$$

If $\{r, s\}$ satisfies (37)-(38) together with the condition

$$
0 \leq 1+3\left(\frac{1}{r_{0}}-\frac{1}{r}\right) \leq 2-j, \quad j=0,1,
$$

then: for all $t>0, j=0,1, \boldsymbol{u} \in \mathbf{L}_{\sigma}^{r_{0}}(\Omega), \nabla \boldsymbol{v} \in \mathbf{L}^{3}(\Omega)$ and $\nabla \boldsymbol{w} \in \mathbf{L}^{m}(\Omega)$,

$$
\begin{aligned}
& \left\|\nabla^{j} e^{-t L}[P(\boldsymbol{u} \cdot \nabla \boldsymbol{v}), \boldsymbol{u} \cdot \nabla \boldsymbol{w}]^{T}\right\|_{\{r, s\}} \\
& \quad \leq C_{\zeta} t^{-(3 / 2)\left(1 / r_{0}-1 / r\right)-(1+j) / 2} e^{-\zeta t}\|\boldsymbol{u}\|_{r_{0}}\left(\|\nabla \boldsymbol{v}\|_{3}+\|\nabla \boldsymbol{w}\|_{m}\right),
\end{aligned}
$$

being $C_{\zeta}=C_{\zeta}\left(r, r_{0}, m, \mu, \mu_{r}, \chi, \gamma\right)$.

Proof: By assumptions, $\{p, q\}$ is such that $1 / p=1 / r_{0}+1 / 3,1 / q=1 / r_{0}+1 / m$. Then (31) implies $p, q \in(1, \infty)$. Moreover, $\{p, q\}$ satisfies (13). We observe that condition (20) is satisfied by $\{p, q\}$; in fact, by assumptions we have

$$
\frac{3}{2}\left(\frac{1}{p}-\frac{1}{r}\right)=\frac{3}{2}\left(\frac{1}{q}-\frac{1}{s}\right)=\frac{1}{2}\left\{1+3\left(\frac{1}{r_{0}}-\frac{1}{r}\right)\right\} \in\left[0,1-\frac{j}{2}\right] .
$$

Therefore, Proposition 4.6 and the Hölder's inequality, implies (41).

Remark 5.4 In $(41)_{j}$, for the integrability at $t=0$, the number $r$ should satisfy $3\left(1 / r_{0}-1 / r\right)<1-j$.

Proof of Proposition 5.1: We consider the following Banach space

$$
\mathcal{B} \equiv\left\{\begin{array}{r}
t^{\beta} e^{\zeta t}(\boldsymbol{u}, \boldsymbol{w}) \in B C\left([0, \infty) ; \mathbf{L}_{\sigma}^{r_{0}}(\Omega) \times \mathbf{L}^{s_{0}}(\Omega)\right) \\
(\boldsymbol{u}, \boldsymbol{w}): t^{1 / 2} e^{\zeta t}(\nabla \boldsymbol{u}, \nabla \boldsymbol{w}) \in B C\left([0, \infty) ; \mathbf{L}^{3}(\Omega) \times \mathbf{L}^{m}(\Omega)\right) \\
\boldsymbol{u}(0)=\boldsymbol{u}_{0}-\overline{\boldsymbol{u}}, \boldsymbol{w}(0)=\boldsymbol{w}_{0}-\overline{\boldsymbol{w}}
\end{array}\right\}
$$

with norm

$$
\left\|[\boldsymbol{u}, \boldsymbol{w}]^{T}\right\|_{\mathcal{B}}=\sup _{t>0} t^{\beta} e^{\zeta t}\left(\|\boldsymbol{u}(t)\|_{r_{0}}+\|\boldsymbol{w}(t)\|_{s_{0}}\right)+\sup _{t>0} t^{1 / 2} e^{\zeta t}\left(\|\nabla \boldsymbol{u}(t)\|_{3}+\|\nabla \boldsymbol{w}(t)\|_{m}\right),
$$


where $s_{0}=\left(\frac{1}{m}+\frac{1}{r_{0}}-\frac{1}{3}\right)^{-1}, \beta=\frac{3}{2}\left(\frac{1}{3}-\frac{1}{r_{0}}\right)=\frac{3}{2}\left(\frac{1}{m}-\frac{1}{s_{0}}\right)>0$. We consider the operator $\Upsilon: \mathcal{B} \rightarrow \mathcal{B}$ given by

$$
\Upsilon\left[\begin{array}{c}
\boldsymbol{u} \\
\boldsymbol{w}
\end{array}\right](t)=e^{-t L}\left[\begin{array}{c}
\boldsymbol{u}_{0}-\overline{\boldsymbol{u}} \\
\boldsymbol{w}_{0}-\overline{\boldsymbol{w}}
\end{array}\right]-\int_{0}^{t} e^{-(t-s) L}\left[\begin{array}{c}
P(\boldsymbol{u} \cdot \nabla \boldsymbol{u}) \\
\boldsymbol{u} \cdot \nabla \boldsymbol{w}
\end{array}\right](s) d s .
$$

For $J>0$, we define the set $\mathcal{B}_{J}=\left\{(\boldsymbol{u}, \boldsymbol{w}) \in \mathcal{B}:\left\|[\boldsymbol{u}, \boldsymbol{w}]^{T}\right\|_{\mathcal{B}} \leq J\right\}$.

We focus the proof on the obtention of bounds for $\Upsilon[\boldsymbol{u}, \boldsymbol{w}]^{T}$ in $\mathcal{B}$. We can take a positive constant $\epsilon=\epsilon\left(r_{0}, m, \mu, \mu_{r}, \gamma, \chi\right)$ such that if $\left\|\boldsymbol{u}_{0}-\overline{\boldsymbol{u}}\right\|_{3}+\| \boldsymbol{w}_{0}-$ $\overline{\boldsymbol{w}} \|_{m}<\epsilon$, there exists a positive constant $J=J\left(\left\|\boldsymbol{u}_{0}-\overline{\boldsymbol{u}}\right\|_{3},\left\|\boldsymbol{w}_{0}-\overline{\boldsymbol{w}}\right\|_{m}\right)$ such that $\Upsilon\left(\mathcal{B}_{J}\right) \subset \mathcal{B}_{J}$, and $\Upsilon$ is contractive on $\mathcal{B}_{J}$.

Since $\gamma+\mu_{r}<\delta^{\prime}, \quad \max \left\{\frac{1}{\mu+\mu_{r}}, \frac{1}{\chi}\right\}<\delta^{\prime}$, we are going to apply the results from Lemmas 5.2 and 5.3 for $j=0,\{r, s\}=\left\{r_{0}, s_{0}\right\}$ and $j=1,\{r, s\}=\{3, m\}$. Indeed, from Lemma 5.2 for $j=0,\{r, s\}=\left\{r_{0}, s_{0}\right\}$, we obtain:

$$
\left\|e^{-t L}\left[\boldsymbol{u}_{0}-\overline{\boldsymbol{u}}, \boldsymbol{w}_{0}-\overline{\boldsymbol{w}}\right]^{T}\right\|_{\left\{r_{0}, s_{0}\right\}} \leq C_{\zeta} t^{-\frac{3}{2}\left(\frac{1}{3}-\frac{1}{r_{0}}\right)} e^{-\zeta t}\left\|\left[\boldsymbol{u}_{0}-\overline{\boldsymbol{u}}, \boldsymbol{w}_{0}-\overline{\boldsymbol{w}}\right]^{T}\right\|_{\{3, m\}},
$$

which is equivalent to

$$
t^{\beta} e^{\zeta t}\left\|e^{-t L}\left[\boldsymbol{u}_{0}-\overline{\boldsymbol{u}}, \boldsymbol{w}_{0}-\overline{\boldsymbol{w}}\right]^{T}\right\|_{\left\{r_{0}, s_{0}\right\}} \leq C_{\zeta}\left\|\left[\boldsymbol{u}_{0}-\overline{\boldsymbol{u}}, \boldsymbol{w}_{0}-\overline{\boldsymbol{w}}\right]^{T}\right\|_{\{3, m\}} .
$$

And from Lemma 5.2 for $j=1,\{r, s\}=\{3, m\}$, we have:

$$
\left\|\nabla e^{-t L}\left[\boldsymbol{u}_{0}-\overline{\boldsymbol{u}}, \boldsymbol{w}_{0}-\overline{\boldsymbol{w}}\right]^{T}\right\|_{\{3, m\}} \leq C_{\zeta} t^{-\frac{1}{2}} e^{-\zeta t}\left\|\left[\boldsymbol{u}_{0}-\overline{\boldsymbol{u}}, \boldsymbol{w}_{0}-\overline{\boldsymbol{w}}\right]^{T}\right\|_{\{3, m\}} .
$$

On the other hand, from Lemma 5.3 for $j=0,\{r, s\}=\left\{r_{0}, s_{0}\right\}$, we obtain:

$$
\begin{aligned}
& \left\|\int_{0}^{t} e^{-(t-s) L}[P(\boldsymbol{u} \cdot \nabla \boldsymbol{u}), \boldsymbol{u} \cdot \nabla \boldsymbol{w}]^{T} d s\right\|_{\left\{r_{0}, s_{0}\right\}} \\
& \quad \leq \int_{0}^{t}\left\|e^{-(t-s) L}[P(\boldsymbol{u} \cdot \nabla \boldsymbol{u}), \boldsymbol{u} \cdot \nabla \boldsymbol{w}]^{T} d s\right\|_{\left\{r_{0}, s_{0}\right\}} d s \\
& \quad \leq C_{\zeta} \int_{0}^{t}(t-s)^{-1 / 2} e^{-\zeta(t-s)}\|\boldsymbol{u}\|_{r_{0}}\left(\|\nabla \boldsymbol{u}\|_{3}+\|\nabla \boldsymbol{w}\|_{m}\right) \\
& \quad \leq C_{\zeta} t^{-\beta} e^{-\zeta t}\left\|[\boldsymbol{u}, \boldsymbol{w}]^{T}\right\|_{\mathcal{B}}^{2} .
\end{aligned}
$$

Analogously, for $j=1,\{r, s\}=\{3, m\}$ we have:

$$
\begin{aligned}
& \left\|\nabla \int_{0}^{t} e^{-(t-s) L}[P(\boldsymbol{u} \cdot \nabla \boldsymbol{u}), \boldsymbol{u} \cdot \nabla \boldsymbol{w}]^{T} d s\right\|_{\{3, m\}} \\
& \leq \int_{0}^{t}\left\|\nabla e^{-(t-s) L}[P(\boldsymbol{u} \cdot \nabla \boldsymbol{u}), \boldsymbol{u} \cdot \nabla \boldsymbol{w}]^{T} d s\right\|_{\{3, m\}} d s \\
& \leq C_{\zeta} \int_{0}^{t}(t-s)^{(-3 / 2)\left(1 / r_{0}-1 / 3\right)-1} e^{-\zeta(t-s)}\|\boldsymbol{u}\|_{r_{0}}\left(\|\nabla \boldsymbol{u}\|_{3}+\|\nabla \boldsymbol{w}\|_{m}\right) d s \\
& \leq C_{\zeta} t^{-1 / 2} e^{-\zeta t}\left\|[\boldsymbol{u}, \boldsymbol{w}]^{T}\right\|_{\mathcal{B}}^{2} .
\end{aligned}
$$


Therefore, from the above inequalities we have

$$
\left\|\Upsilon[\boldsymbol{u}, \boldsymbol{w}]^{T}\right\|_{\mathcal{B}} \leq C_{0}\left\|\left[\boldsymbol{u}_{0}-\overline{\boldsymbol{u}}, \boldsymbol{w}_{0}-\overline{\boldsymbol{w}}\right]^{T}\right\|_{\{3, m\}}+C_{1}\left\|[\boldsymbol{u}, \boldsymbol{w}]^{T}\right\|_{\mathcal{B}}^{2} .
$$

So, taking $J_{0}=C_{0}\left\|\left[\boldsymbol{u}_{0}-\overline{\boldsymbol{u}}, \boldsymbol{w}_{0}-\overline{\boldsymbol{w}}\right]^{T}\right\|_{\{3, m\}}<\frac{1}{4 C_{1}}$, we get:

$$
\Upsilon\left(\mathcal{B}_{J}\right) \subset\left(\mathcal{B}_{J}\right), \text { with } J=\frac{1-\sqrt{1-4 C_{1} J_{0}}}{2 C_{1}} .
$$

Working in a similar manner, we can show that $\Upsilon$ is contractive. Consequently, the system (30) has a solution in $\mathcal{B}_{J}$, that satisfies the estimates (32)-(33) for $\{r, s\}=\left\{r_{0}, s_{0}\right\}$ and the estimates (34)-(35) for $\left\{r_{1}, s_{1}\right\}=\{3, m\}$.

The uniqueness proof is done in a standard way, so it is omitted. To see that the solution $(\widetilde{\boldsymbol{u}}, \widetilde{\boldsymbol{w}})$ of (30) satisfies the estimates (32)-(33) for the other values of $r, s, r_{1}, s_{1}$, we use again Lemma 5.2 and Lemma 5.3. Using arguments analogous of those of Fujita \& Kato [7], Giga \& Miyakawa [9], Kozono \& Ogawa [13], we can show the Hölder continuity with respect to time for the nonlinear term in $\mathbf{L}_{\sigma}^{3}(\Omega) \times \mathbf{L}^{\alpha}(\Omega)$ and this allows us to show that $(\widetilde{\boldsymbol{u}}, \widetilde{\boldsymbol{w}})$ is, in fact, a strong solution for the system (29) in the sense given in Definition 2.3 (see Tanabe [29]), Theorem 3.3.4).

Proof of Theorem 2.4: We take $\alpha=m=3 / 2$ and we choose $r_{0}(m)$ satisfying (31). If $\chi+\mu_{r}<\delta^{\prime}, \quad \max \left\{\frac{1}{\mu+\mu_{r}}, \frac{1}{\chi}\right\}<\delta^{\prime}$, then by Proposition 5.1 , there exists a positive constant $\epsilon=\epsilon\left(\mu, \mu_{r}, \gamma, \chi, m, r_{0}\right)$ such that if $\| \boldsymbol{u}_{0}-$ $\overline{\boldsymbol{u}}\left\|_{3}+\right\| \boldsymbol{w}_{0}-\overline{\boldsymbol{w}} \|_{m}<\epsilon$, there exists a unique strong solution $(\widetilde{\boldsymbol{u}}, \widetilde{\boldsymbol{w}})$ for system (29) in the interval $[0, \infty)$. Therefore, $(\widetilde{\boldsymbol{u}}, \widetilde{\boldsymbol{w}})$ satisfies the estimates (32)-(35), being $\zeta>0$ a number positive fix that satisfies $(26)$ for $\{p, q\}=\left\{3 r_{0} /(3+\right.$ $\left.\left.r_{0}\right), m r_{0} /\left(m+r_{0}\right)\right\}$ and $\{3, m\}$.

¿From Nirenberg's inequality and using the estimate for $\|\nabla \widetilde{\boldsymbol{u}}(t)\|_{r^{\prime}}$ for some $r^{\prime}>3$, we obtain

$$
\|\widetilde{\boldsymbol{u}}(t)\|_{\infty} \leq C\|\nabla \widetilde{\boldsymbol{u}}(t)\|_{r^{\prime}}^{a}\|\widetilde{\boldsymbol{u}}(t)\|_{3}^{(1-a)} \leq C_{\zeta} \widetilde{C} t^{-(3 a / 2)\left(1 / 3-1 / r^{\prime}\right)-a / 2} e^{-\zeta t},
$$

where $a$ satisfies $0=a\left(1 / r^{\prime}-1 / 3\right)+(1-a) 1 / 3$. This implies that $\|\overline{\boldsymbol{u}}(t)\|_{\infty}=$ $o\left(e^{-\zeta t}\right)$, when $t \rightarrow \infty$.

Observe that we cannot apply the above argument to find the decay rate for the microrotation velocity $\boldsymbol{w}$. In fact, in this case for $m=3 / 2, s_{0}=\left(\frac{1}{m}+\frac{1}{r_{0}}-\right.$ $\left.\frac{1}{3}\right)^{-1}<3$. To overcome this point, we apply Proposition 5.1 with another value for $m$. In fact, we choose $m_{0}$ and $r_{0}$ such that $\frac{3}{2}<\frac{3 r_{0}}{2 r_{0}-3}<m_{0}<3$. The above inequality implies that $1 / m_{0}+1 / r_{0}<2 / 3$, that is,

$$
\left(\frac{1}{m_{0}}+\frac{1}{r_{0}}-\frac{1}{3}\right)^{-1}>3 \text {. }
$$

From the estimates (32)-(35) with $\{r, s\}=\left\{3, m_{0}\right\}$, we can find a time $T>0$ big enough such that

$$
\|\widetilde{\boldsymbol{u}}(T)\|_{3}+\|\widetilde{\boldsymbol{w}}(T)\|_{m_{0}}<\epsilon\left(\mu, \mu_{r}, \chi, \gamma, m_{0}, r_{0}\right),
$$


provided that $\gamma+\mu_{r}<\delta^{\prime}\left(m_{0}, r_{0}\right), \max \left\{\frac{1}{\mu+\mu_{r}}, \frac{1}{\chi}\right\}<\delta^{\prime}\left(m_{0}, r_{0}\right)$, with

$$
\widetilde{\delta}^{\prime}\left(m_{0}, r_{0}\right) \equiv \min \left\{\delta_{3 r_{0} /\left(3+r_{0}\right), m_{0} r_{0} /\left(m_{0}+r_{0}\right)}, \delta_{3, m_{0}}\right\} .
$$

Let $\delta=\min \left\{\widetilde{\delta}^{\prime}\left(m_{0}, r_{0}\right), \delta^{\prime}\left(m, r_{0}\right)\right\}$, where $\delta^{\prime}$ is given as before and we assume:

$$
\gamma+\mu_{r}<\delta, \quad \max \left\{\frac{1}{\mu+\mu_{r}}, \frac{1}{\chi}\right\}<\delta .
$$

Since $(\boldsymbol{u}(T), \boldsymbol{w}(T)) \in \mathbf{L}_{\sigma}^{3}(\Omega) \times \mathbf{L}^{m_{0}}(\Omega)$, we apply Proposition 5.1 with initial condition equal to $(\boldsymbol{u}(T), \boldsymbol{w}(T))$ and $m=m_{0}$. Consequently, we can deduce (32)-(35) and $m=m_{0}$, with $\zeta>0$ a fixed positive number satisfying (26) for $\{p, q\}=\left\{3 r_{0} /\left(3+r_{0}\right), m_{0} r_{0} /\left(m_{0}+r_{0}\right)\right\}$ and $\left\{3, m_{0}\right\}$.

Now, we can obtain the decay rates of $\|\nabla \widetilde{\boldsymbol{w}}(t)\|_{s}$ for some $s>3$ because (42) is verified. We observe that in this point we have used the uniqueness of solution. Consequently, we have $\|\widetilde{\boldsymbol{w}}(t)\|_{\infty}=o\left(e^{-\zeta t}\right)$, when $t \rightarrow \infty$.

\section{Acknowledgments:}

The authors would like to express their gratitude to one of the referees for his/her interesting suggestions and comments.

\section{References}

[1] W. Borchers, T. Miyakawa, On stability of exterior stationary NavierStokes flows. Acta Math. 174(2), (1995) 311-382.

[2] Z. M. Chen, $L^{r}$ - stability of viscous incompressible fluid motions in unbounded domains. J. Math. Anal. Appl. 181(3), (1994) 768-788.

[3] D. W. Condiff, J. S. Dahler, Fluid mechanics aspects of antisymmetric stress. Phys. Fluids, 7 (1964) 842-854.

[4] A. C. Eringen, Simple microfluids, Int. J. Enging. Sci. 2 (1964) 205-217.

[5] A. C. Eringen, Theory of micropolar fluids, J. Math. Mech. 16 (1966) 1-8.

[6] D. Fujiwara, H. Morimoto, An $L^{r}$-Theorem of the Helmholtz decomposition of vectos fields. Fac. Sci. Univ. Tokio, sec. IA. 24(3), (1977) 685-700.

[7] H. Fujita, T. Kato, On the Navier-Stokes initial value-problem, I. Arch. Rational Mech. Anal. 16 (1964) 269-315.

[8] Y. Giga, Analiticy of the semigroup generated by the Stokes operator in $L_{r}$ spaces. Math. Z. 178(3), (1981) 297-329.

[9] Y. Giga, T. Miyakawa, Solutions in $L_{r}$ of the Navier-Stokes initial value problem. Arch. Rational Mech. Anal. 89(3), (1985) 267-281. 
[10] Y. Giga, Domains of fractional powers of the Stokes operator in $L_{r}$ spaces. Arch. Rational Mech. Anal. 89(3), (1985) 251-265.

[11] T. Hishida, Global existence and exponential stability of convection. J. Math. Anal. Appl. 196, (1995) 699-721.

[12] T. Hishida, On a class of stable steady flow to the exterior convection problem. J. Diff. Eqns. 141, (1997) 54-85.

[13] H. Kozono, T. Ogawa, Some $L^{p}$ estimate for the exterior Stokes flow and an application to the nonstationary Navier-Stokes equations. Indiana Univ. Math. J. 41(3), (1992) 789-808.

[14] H. Kozono, T. Ogawa, On stability of the Navier-Stokes flows in exterior domains. Arch. Rational Mech. Anal. 128(1), (1994) 1-31.

[15] H. Kozono, T. Ozawa, Stability in $L^{r}$ of the Navier-Stokes flows in an n-dimensional bounded domain. J. Math. Anal. Appl. 152(1),(1990) 3545 .

[16] H. Kozono, M. Yamazaki, On a larger class of stable solutions to the NavierStokes equations in exterior domains. Math. Z. 228, (1998) 751-785.

[17] O. Ladyszhenskaya, The Mathematical Theory of Viscous Incompressible Flow, second edition, Gordon and Breach Eds. New York, 1969.

[18] G. Lukaszewicz, On stationary flows of asymmetric fluids, Rend. Accad. Naz. Sci. XL Mem. Mat.(5), 12(1), (1988) 35-44.

[19] G. Lukaszewicz, On nonstationary flows of asymmetric fluids, Rend. Accad. Naz. Sci. XL Mem. Mat.(5), 12(1),(1988) 83-97.

[20] G. Lukaszewicz, On the existence, uniqueness and asymptotic properties for solutions of flows of asymmetric fluids, Rend. Accad. Naz. Sci. XL Mem. Mat.(5), 13(1)(1989) 105-120.

[21] G. Lukaszewicz, Micropolar Fluids. Theory and Applications, Modelling and Simulation in Science, Engineering and Technology. Birkäuser, Boston, Basel, Berlin, 1999.

[22] E. Ortega-Torres, M. Rojas-Medar, On the uniqueness and regularity of the weak solutions for magneto-micropolar equations. Rev. Mat. Apl. 17(2)(1996) 75-90.

[23] E. E. Ortega-Torres, M. A. Rojas-Medar, The equations of a viscous asymmetric fluids: an iterative approach. Z. Angew. Math.Mech. 85(7), (2005) 471-489.

[24] A. Pazy, Semigroups of Linear Operators and Applications to Partial differential Equations. Applied Math. Sci., 44, Springer, 1983. 
[25] L. Petrosyan, Some Problems of Fluids Mechanics with Antisymmetric Stress Tensor. Erevan, 1984 (in Russian).

[26] M. Rojas-Medar, J. L. Boldrini, Magneto-micropolar fluid motion: existence of weak solution. Rev. Mat. Univ. Complutense de Madrid, 11(2),(1998) 443-460.

[27] M. Rojas-Medar, Magneto-micropolar fluid motion: existence and uniqueness of strong solution. Math. Nachr. 188,(1997) 301-319.

[28] M. Rojas-Medar, Magneto-micropolar fluid motion: on the convergence rate of the spectral Galerkin approximations. Z. Angew. Math. Mech. 77(10),(1997) 723-732.

[29] H. Tanabe, Equations of evolution. Pitman, London, 1979.

[30] R. Temam, Navier-Stokes Equations, Theory and Numerical Analysis, North - Holland (2 ${ }^{\text {nd }}$ Revised Edition). Amsterdam, 1979.

[31] N. Yamaguchi, Existence of global solution to the micropolar fluid system in bounded domain. Math. Meth. Appl. Sci. 28 (13),(2005) 1507-1526. DOI:10.1002/mma.617

Elder Jesús Villamizar-Roa

Escuela de Matemáticas

Universidad Industrial de Santander-UIS

A.A. 678 , Bucaramanga-Santander

Colombia

e-mail: jvillami@uis.edu.co

María Ángeles Rodríguez-Bellido

Dpto. Ecuaciones Diferenciales y Análisis Numérico,

Universidad de Sevilla

Aptdo. 1160, 41080 Sevilla

Spain

e-mail: angeles@us.es 